\title{
"Trail" in Cancers - Time to Curb the Crab: A Meta-Analysis Research
}

\section{Ayushi Bhargava ${ }^{1}$ and Gaurav ${ }^{2 *}$}

${ }^{1}$ House Surgeons, NSVK Sri Venkateshwara Dental College and Hospital, Bangalore, Karnataka, India

${ }^{2}$ Consultant Oral Physician and Maxillofacial Radiologist, Assistant Professor,

Department of Oral Medicine and Maxillofacial Radiology, NSVK Sri

Venkateshwara Dental College and Hospital, Bangalore, Karnataka, India

*Corresponding Author: Gaurav, Consultant Oral Physician and Maxillofacial

Radiologist, Assistant Professor, Department of Oral Medicine and Maxillofacial

Radiology, NSVK Sri Venkateshwara Dental College and Hospital, Bangalore,

Karnataka, India.
Received: February 25, 2021

Published: March 20, 2021

(C) All rights are reserved by Ayushi Bhargava and Gaurav.

\section{Abstract}

Background: TRAIL (tumor necrosis factor related apoptosis inducing ligand) is the one of the recently developed method for curing cancer. The method demonstrates modified lipid nanoparticles which attaches to TRAIL and E selectin all of which coats the WBCs. The designed nature of modified leukocytes is cytotoxic and shows homology to TNF leading to targeted death of cancerous cells without harming normal healthy cells of one's body. These modified leukocytes designed using body's own defenses track down and induces apoptosis of absconding cancer cells. This approach shows large potential for patients with metastatic cancer as TRAIL coated leukocytes occur innate. Since current therapies have lot of disadvantages with it, which allows TRAIL therapy to be investigated at a large scale.

Aim of the Study: To determine the significance of TRAIL and modified Leukocytes in inducing the inhibition of tumor genesis.

Research Hypothesis: Is TRAIL actually significant in inducing the inhibition of tumor genesis?

Materials and Methodology: With the medicine database taken as a source for authenticated scientific research data, articles were selected having undergone Randomized Control Trail. Out of these, articles (studies) were chosen which met the criterion for Metaanalysis.

Result and Conclusion: From the study and forest plot interpretation it demonstrates that the study is significant and which manifest that TRAIL does have higher sensitivity and specificity in inducing inhibition of tumor genesis.

Keywords: Cancer; TRAIL (Tumor Necrosis Factor Related Apoptosis Inducing Ligand); Leukocytes

\section{Introduction}

Cancer is the second leading cause of death globally, in 2018 around 9.6million people worldwide were estimated to have died from cancer as stated by "World Health Organization". Cancer can develop in any of the body's tissues, and each type of cancer has its own features, but the basic processes that cause cancer are almost similar [27]. This similarity can help to devise common methodology for the treatment of it. Typically, the current standards of treatment have a downside to it, which is it has an unspecified mode of action often causing substantial death of healthy cells. TRAIL has 
been the limelight in research process as it can specifically and robustly target cancerous cells.

TRAIL is an acronym which stands for Tumor necrosis factor related apoptosis inducing ligand. It is an immune cytokine of Tumor Necrosis Factor super family and there are 27 family members numerically classified as TNFRSF\# in which TRAIL is type 10, it is generated and secreted by almost all normal tissue cells. It has also been known as CD253 and TNFSF10 [5,7].

In human beings, the gene present on chromosome 3q26 encodes for TRAIL. Genomic structure of TRAIL consists of five exonic segments and spans approximately $20 \mathrm{~kb}$.

Its function which attracts the field of cancer therapeutics is that it can initiate apoptosis through the activation of death receptors- DR4 and DR5, resulting in selective induction of apoptosis in cancerous cells $[13,14]$.

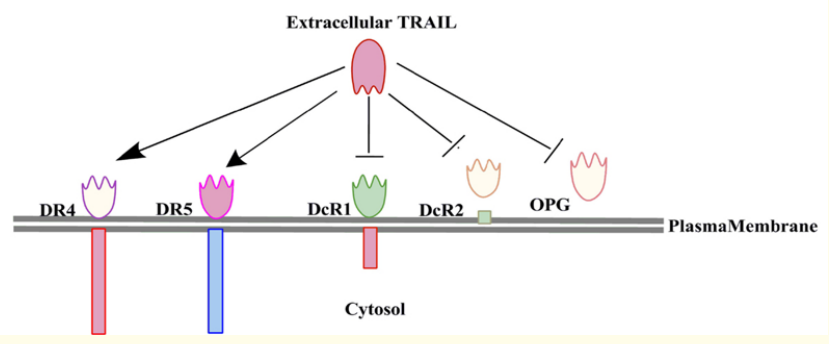

Figure 1: Extracellular TRAIL attaching to DR4 and DR5.

\section{Aim of the Study}

To determine the significance of TRAIL and modified Leukocytes in inducing the inhibition of tumor genesis.

\section{Research Question}

Is TRAIL actually significant in inducing the inhibition of tumor genesis?

\section{Materials and Methods}

Various research and studies have documented the significance of TRAIL for the treatment method of cancer. With this certitude, a literature-based Meta-analysis research was carried out to ac- complish the aim of the study. Along with the concepts and fundamentals as preamble, Department of Oral Medicine and Radiology designed meta-analysis research. With Cochrane collaboration and other scientific research databases like Medline and Medknow were taken as authenticated scientific research sources, about 35 research articles having undergone Randomized Control Trial were chosen out of which 17 articles finally met criterion for metaanalysis.

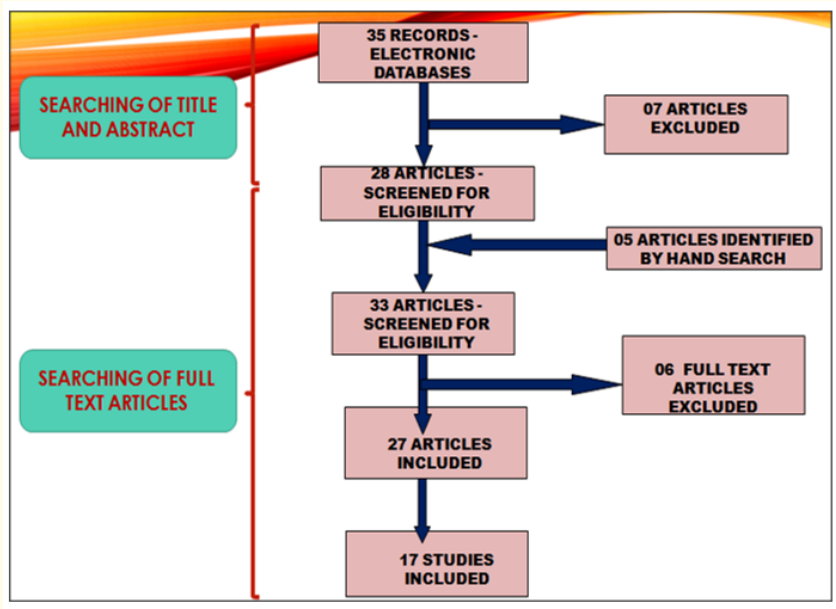

Figure 2: Methodology and chronology of inclusion and exclusion of articles.

\section{Result}

The statistical analysis was determined in the form of Forest plot. Interpreting the graph, there is odds ratio and standard deviation values for each article. The selected articles were subjected to thorough understanding to account overall probability value ( $p$ value) of articles. After the $p$ value or the probability value of each of these articles were subjected and tabulated to statistical analysis using V5 software we obtain a graph which is known as forest plot. Interpreting this graph, we see on left hand side all 17 articles are included in our study. On extreme right we have all these standard deviation values of each of these articles taken into consideration.

A dark vertical central line shows the null hypothesis line or median line and the horizontal line against each of these articles represents significance of these articles in the form of box shaped 
square. However, taking submission or culmination and average of all the $\mathrm{p}$ values taken into consideration we obtain a diamond shaped box which is present at the bottom of null hypothesis line which lies right to it demonstrating that our study is a significant one and with p-value of 0.0001 .

So, finally interpreting the result from the above-mentioned forest plot and using other methodologies it can be interpreted that TRAIL does have higher sensitivity and specificity towards combating the reoccurrence and metastasis rate of cancer.

It can potentially be dwelled into the new era of cancer diagnostics and therapeutics.

Thus, through thoroughly accessing the synchronization of TRAIL apoptosis pathway, it can help to develop more selective TRAIL - based agents for the cure of human cancer [16].

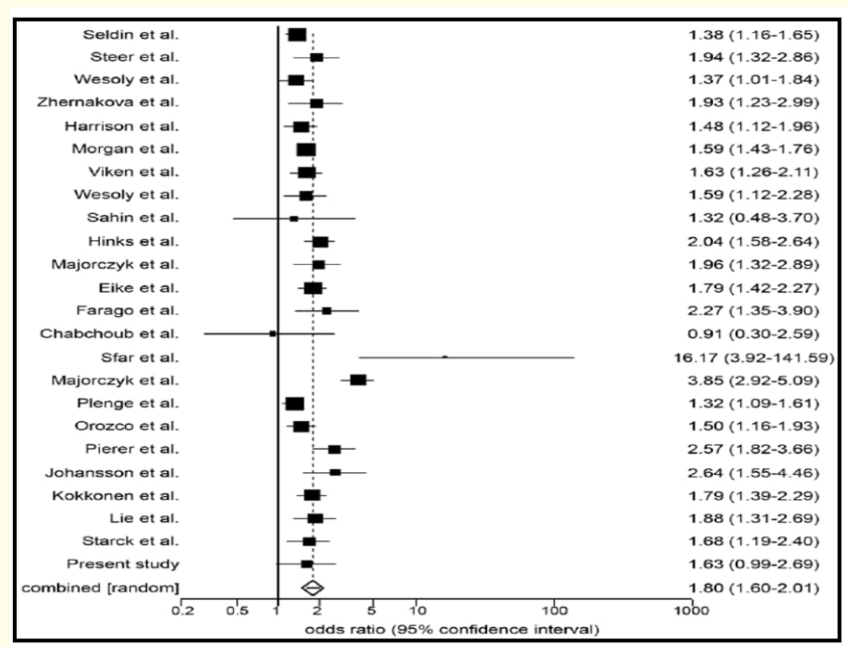

Graph 1: Forest plot (meta-analysis).

\section{Discussion}

Tumor necrosis factor related apoptosis inducing ligand therapy was released as an untagged soluble human TRAIL which had short serum half-life so; to increase its solubility it was attached to leukocytes through e-selectin. Now these modified leukocytes act as modified ligands which are cytotoxic in nature and shows homology to the TNF leading targeted death. It can bind to cell surface death receptors, TRAIL-R1 (DR4) and TRAIL-R2 (DR5) and helps in formation of death inducing signaling complex, eventually activating p53-independent apoptotic cascade. This mechanism makes TRAIL a potential anti-cancer therapy especially for p53 mutated tumors $[8,10]$.

It generally follows 2 signaling pathways; these are mitochondrial independent activation of caspase 3 and mitochondrial dependent apoptosis by caspase 8. TRAIL defined apoptosis is caspase-8-dependent which is an initiator caspase. Caspase 8 cleaves inactive effector caspases which consists of procaspase 3, 6 and 7 leading to activation of kinases causing cascade reaction which triggers the apoptotic process $[17,19]$.

Another method of TRAIL to induce apoptosis is that it can also bind to DcR1 and Dcr2 but it does consist of cytoplasmic domain (DcR1) or truncated death domain (DcR2). DcR1 has a function of neutralizing TRAIL receptors. The cytoplasmic domain of DcR2 activates NFkappaB. Activated NFkappaB leads to transcription of genes which finally breakthrough to death signaling pathway $[18,22]$.

However, recombinant human TRAIL protein (rhTRAIL) and TRAIL-R agonist monoclonal antibodies can be not successful to exert anticancer activities due to various reasons like inherent or acquired resistance, weak potencies, and poor pharmacokinetics for apoptosis induction. Because of these reasons some patients can show resistance to TRAIL induced apoptosis, for which evaluation of tumor DR4 and FLIP expression and host DR4 codon441 could be useful predictor of TRAIL sensitivity [26,29].

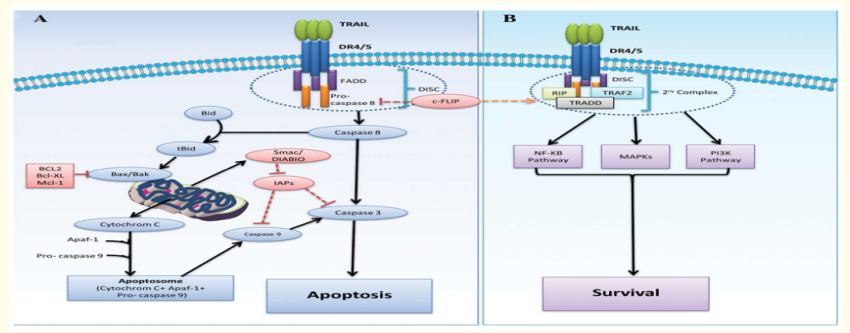

Figure 3: A: Signaling pathways of TRAIL to induce apoptosis, B: Resistant shown towards TRAIL pathway. 
TRAIL- TRAIL-R binding can cause non apoptotic signaling through activation of p38, ERK, RAC1, nuclear factor- kB. But patients with immunosuppressive cancer might get advantage from TRAIL-R therapy. To modify the coming drawbacks multiple strategies including combinatorial approach, protein modification and TRAIL gene therapy are being extensively explored. These methods aim to enhance half-life and bioavailability of TRAIL and show coactions with TRAIL ultimately sensitizing the resistant cells [21,28].

The possible promising outcomes of TRAIL therapy makes the clinicians more interested in search of more specific targeted therapy related to it.
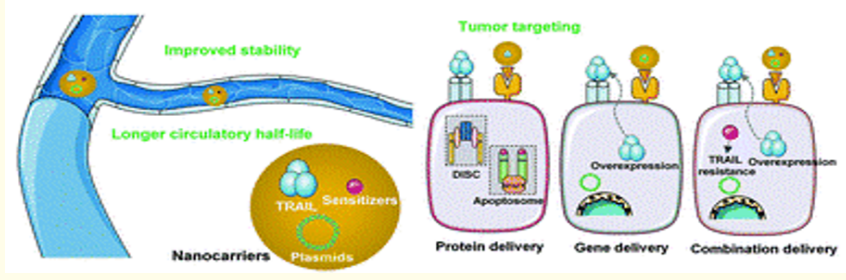

Figure 4: Modified therapies adopted to overcome drawbacks of basic TRAIL therapy.

\section{Conclusion}

The modern medicine has aced to achieve cure for almost all the aliments affecting the mankind but still the section of cancer is an ongoing process in the field of therapeutics. Till now current methodologies are having down streaming affects which makes the need of TRAIL a key highlight of need in the modern world evoking the interest to study pro-apoptotic TRAIL receptors and signaling pathways [11].

TRAIL- tumor necrosis factor inducing ligand is a member of TNF super family which is an immune cytokine. Main function which is the epitome of TRAIL is its ability to induce apoptosis specifically targeting cancerous cells not causing any aliment to other normal tissues which is a major drawback of chemotherapy which is the one of the major therapeutics to cure cancer $[13,15]$.

However, under many conditions patients can show resistance to this therapy leading to the need of getting TRAIL back on track as cancer therapeutics. Many modifications and strategies are adapted to overcome these drawbacks like engineered ligands, protein modification and TRAIL gene therapy etc [4,32].

The future prospects and possibilities of the TRAIL therapy holds promising outcomes, as mentioned above and by interpreting the forest plot it proves that sensitivity and specificity of TRAIL therapy has higher rate in combating the metastasis of cancer. More research in this area of therapy can be favorable for the tremendously burdened patients and can serve as a boon to mankind. And moreover, there is a whole new arena yet to be explored and being benefitted from TRAIL therapy, which can be a breakthrough in the world of oncology medicine and therapeutics.

\section{Bibliography}

1. Seldin DC., et al. "Tolerability and efficacy of thalidomide for the treatment of patients with light chain-associated (AL) amyloidosis (2003).

2. Signal Integration of IFN-I and IFN-II With TLR4 Involves Sequential Recruitment of STAT1-Complexes and NFkB to Enhance Pro-inflammatory TranscriptionData_Sheet_1.PDFData_Sheet_2.ZIP.

3. Fanger NA., et al. "Human dendritic cells mediate cellular apoptosis via tumor necrosis factor-related apoptosis-inducing ligand (TRAIL)". Journal of Experimental Medicine 190.8 (1999): 1155-1164.

4. Hellwig CT and Rehm M. "TRAIL signaling and synergy mechanisms used in TRAIL-based combination therapies". Molecular Cancer Therapeutics 11 (2012): 3-13.

5. Von Karstedt, S., et al. "Exploring the TRAILs less travelled: TRAIL in cancer biology and therapy". Nature 17 (2017): 352366.

6. Kayagaki N., et al. "Involvement of TNF-related apoptosisinducing ligand in human CD4+ T cell-mediated cytotoxicity". Journal of Immunology 162.5 (1999): 2639-2647.

7. Loss of TRAIL-Receptors Is a Recurrent Feature in Pancreatic Cancer and Determines the Prognosis of Patients with No Nodal Metastasis after Surgery (2013). 
8. Griffith TS., et al. "Monocyte-mediated tumoricidal activity via the tumor necrosis factor-related cytokine, TRAIL". Journal of Experimental Medicine 189.8 (1999): 1343-1354.

9. Wajant H., et al. "TNF-related apoptosis inducing ligand (TRAIL) and its receptors in tumor surveillance and cancer therapy". Apoptosis 7.5 (2002): 449-459.

10. Muhlenbeck F, et al. "The tumor necrosis factor-related apoptosis-inducing ligand receptors TRAIL-R1 and TRAIL-R2 have distinct cross-linking requirements for initiation of apoptosis and are non-redundant in JNK activation". Journal of Biological Chemistry 275.41 (2000): 32208-32213.

11. Kim K., et al. "Molecular determinants of response to TRAIL in killing of normal and cancer cells". Clinical Cancer Research 6.2 (2000): 335-346.

12. MacFarlane M., et al. "'Identification and molecular cloning of two novel receptors for the cytotoxic ligand TRAIL". Journal of Biological Chemistry 272.41 (1997): 25417-25420.

13. Spierings DC., et al. "Tissue distribution of the death ligand TRAIL and its receptors". Journal of Histochemistry and Cytochemistry 52.6 (2004): 821-831.

14. Emery JG., et al. "Osteoprotegerin is a receptor for the cytotoxic ligand TRAIL (2020).

15. Sanlioglu AD., et al. "Differential expression of TRAIL and its receptors in benign and malignant prostate tissues". Journal of Urology 177.1 (2007): 359-364.

16. Daniels RA., et al. "Expression of TRAIL and TRAIL receptors in normal and malignant tissues". Cell Research 15.6 (2005): 430-438.

17. Henry CM and Martin SJ. "Caspase-8 Acts in a Non-enzymatic Role as a Scaffold for Assembly of a Pro-inflammatory "FADDosome“ Complex upon TRAIL Stimulation". Molecular Cell 65 (2017): 715-729.

18. Van Noesel MM., et al. "Tumor-specific down-regulation of the tumor necrosis factor-related apoptosis-inducing ligand decoy receptors DcR1 and DcR2 is associated with dense promoter hypermethylation". Cancer Research 62.7 (2002): 2157-2161.
19. Macfarlane M., et al. "Identification and molecular cloning of two novel receptors for the cytotoxic ligand TRAIL". The Journal of Biological Chemistry 272.41 (1997): 25417-25420.

20. Truneh A., et al. "Temperature-sensitive differential affinity of TRAIL for its receptors. DR5 is the highest affinity receptor". The Journal of Biological Chemistry 275.30 (2000): 233192325.

21. McCourt C., et al. "Elevation of c-FLIP in Castrate-Resistant Prostate Cancer Antagonizes Therapeutic Response to Androgen Receptor-Targeted Therapy". Clinical Cancer Research 18 (2012): 3822-3833.

22. Bernard D., et al. "Rel/NF-kappaB transcription factors protect against tumor necrosis factor (TNF)-related apoptosis-inducing ligand (TRAIL)-induced apoptosis by up-regulating the TRAIL decoy receptor DcR1". The Journal of Biological Chemistry 276.29 (2001): 27322-27328.

23. Clancy L., et al. "Preligand assembly domain-mediated ligandindependent association between TRAIL receptor 4 (TR4) and TR2 regulates TRAIL-induced apoptosis". Proceedings of the National Academy of Sciences of the United States of America 102 (2005): 18099-18104.

24. Sheridan JP., et al. "Control of TRAIL-induced apoptosis by a family of signaling and decoy receptors". Science 277.5327 (1997): 818-821.

25. Marsters SA., et al. "A novel receptor for Apo2L/TRAIL contains a truncated death domain". Current Biology 7.12 (1997): 1003-1006.

26. Wolkers MC., et al. "Nab2 regulates secondary CD8+ T-cell responses through control of TRAIL expression". Blood 119 (2012): 798-804.

27. World Health Organization!. "Bibliography on the epidemiology of cancer, 1946-1960 = Bibliographie de l' épidémiologie du cancer, 1946-1960". Genève: World Health Organization (1963).

28. Ikeda T., et al. "Dual Effects of TRAIL in Suppression of Autoimmunity: The Inhibition of Th1 Cells and the Promotion of Regulatory T Cells". Journal of Immunology 185 (2010): 52595267. 
29. Clancy L., et al. "Preligand assembly domain-mediated ligandindependent association between TRAIL receptor 4 (TR4) and TR2 regulates TRAIL-induced apoptosis". Proceedings of the National Academy of Sciences of the United States of America 102.50 (2005): 18099-18104.

30. Dicker F., et al. "Fas-ligand (CD178) and TRAIL synergistically induce apoptosis of CD40-activated chronic lymphocytic leukemia B cells". Blood 105 (2005): 3193-3198.

31. Dorothee G., et al. "Tumor-Infiltrating CD4+ T Lymphocytes Express APO2 Ligand (APO2L)/TRAIL upon Specific Stimulation with Autologous Lung Carcinoma Cells: Role of IFN- $\alpha$ on APO2L/TRAIL Expression and -Mediated Cytotoxicity". Journal of Immunology 169.2 (2002): 809-817.

32. Chuster IS., et al. "TRAIL+ NK Cells Control CD4+ T Cell Responses during Chronic Viral Infection to Limit Autoimmunity". Immunity 41 (2014): 646-656.

33. Radaeva S., et al. "Natural Killer Cells Ameliorate Liver Fibrosis by Killing Activated Stellate Cells in NKG2D-Dependent and Tumor Necrosis Factor-Related Apoptosis-Inducing LigandDependent Manners". Gastroenterology 130 (2006): 435-452.

34. Cuff AO., et al. "The obese liver environment mediates conversion of NK cells to a less cytotoxic ILC1-like phenotype". Frontiers in Immunology 10 (2019): 2180.

35. Weckmann M., et al. "Critical link between TRAIL and CCL20 for the activation of $\mathrm{TH} 2$ cells and the expression of allergic airway disease". Nature Medicine 13 (2007): 1308-1315.

\section{Assets from publication with us}

- Prompt Acknowledgement after receiving the article

- Thorough Double blinded peer review

- Rapid Publication

- Issue of Publication Certificate

- High visibility of your Published work

Website: www.actascientific.com/

Submit Article: www.actascientific.com/submission.php

Email us: editor@actascientific.com

Contact us: +919182824667 